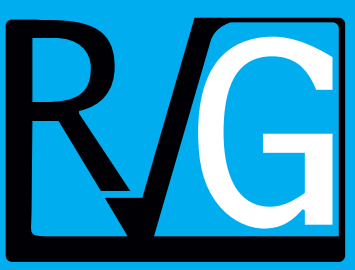

Año 21 No. 74

Abril - Junio 2016

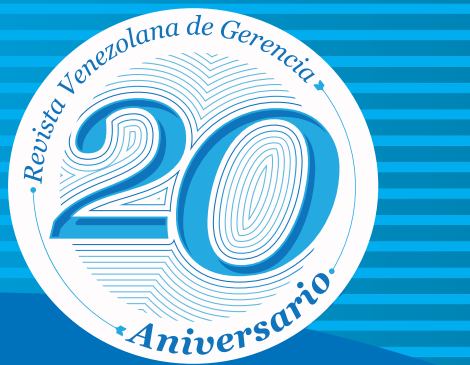

Venezolana de
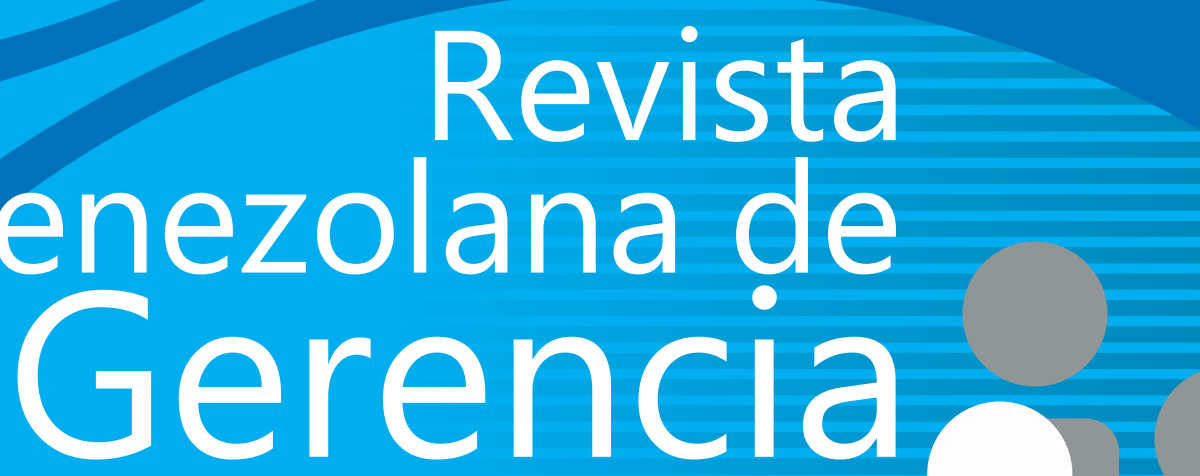


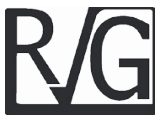

\title{
Factores socio-culturales asociados al emprendedor: evidencia empírica para América Latina
}

\author{
Martínez Gámez, Angel Enrique*
}

\section{Resumen}

En Latinoamérica no es abundante la literatura que estudia el emprendimiento y los factores asociados a esta actividad. Surge la importancia del análisis de la figura del emprendedor y sus determinantes, con la finalidad de establecer sus rasgos sociales, estímulos y principales obstáculos a la hora de poner en marcha su propio negocio. El objetivo del presente documento consiste en describir y analizar algunos factores socio-culturales que influyen en la decisión de emprender una actividad económica en América Latina. Con datos de la encuesta de la Corporación Andina de Fomento para el año 2012 proveniente de 9 países, se especifican aptitudes para el emprendimiento junto con otras series de variables que permiten categorizar a las personas involucradas en la actividad emprendedora. A través de ello, se aplicó un modelo logit para determinar las relaciones existentes de diversas variables en el emprendimiento individual. Los resultados muestran principalmente relación positiva entre el emprendedor y la habilidad empresarial, la experiencia, el nivel educativo, tradición familiar, el número de hijos y la condición de estar casado o en unión. El emprendedor es el resultado de interacciones sociales y de características propias como las habilidades empresariales, las cuales pueden ser adquiridas y potenciadas mediante la experiencia y la formación.

Palabras clave: emprendimiento; trabajo independiente; capital humano.

Recibido: 15.02.16 Aceptado: 20.05.16

* Doctorando en Ciencias Económicas, Magister en Desarrollo Económico y Magister Scientiarum en Moneda e Instituciones Financieras, Departamento de Ciencias Económicas y Administrativas, Universidad Simón Bolívar, Caracas - Venezuela. E-mail: angelmartinez@usb.ve 


\title{
Socio-cultural factors associated with the entrepreneur: empirical evidence for Latin America
}

\begin{abstract}
In Latin America, the literature about entrepreneurship, and its factors, is scarce. The importance of the analysis of the entrepreneur figure and its determinants shows up, in order to establish their social features, stimuli and the main obstacles when organizing a self-owned business. The objective of the present document is to study some socio-cultural factors that influence the decision to undertake an economic activity in Latin America. The data from the 2012 CAF survey, from 9 countries, specifies skills for entrepreneurship along side with other sets of variables which allow you to categorize the people involved in entrepreneurial activity. Through it, a logit econometric model was applied to determine the relationships of different variables on the individual entrepreneurship. Results show a positive relationship between entrepreneur and entrepreneurial skill, experience, education level, family tradition, the number of children and the condition of being married.
\end{abstract}

Key words: entrepreneurship; self-employment; human capital.

\section{Introducción}

El desempleo y la escasez de oportunidades de trabajo asalariado presente en la mayoría de los países de Latinoamérica conllevan a los individuos a la búsqueda de alternativas para mejorar su situación económica. El emprendimiento surge como una de esas alternativas en pro de mejorar su situación particular y así contribuir con las economías de la región. Sin embargo, la evidencia sugiere que las tres cuartas partes de los microempresarios de la región poseen condición de emprendedores de subsistencia de baja productividad (CAF, 2013:113).

Una de las razones para el estudio del emprendimiento se basa en el supuesto de que influye en el crecimiento económico de los países, diversifica la actividad económica, genera ingresos complementarios, movilidad social, entre otros (Valliere y Peterson, 2009:460-461; Kwon y Arenius, 2010:316). Por lo tanto, es de vital importancia el análisis de la figura del emprendedor con la finalidad de establecer sus rasgos sociales, estímulos y los principales obstáculos a la hora de poner en marcha su propio negocio.

Es evidente que el fomento del emprendimiento ha sido una política de empleo en la mayoría de los países por considerarse motor de la innovación, la competitividad y el crecimiento. Sin embargo, el potencial emprendedor muchas veces deja de ser una solución para convertirse en una problemática por diversos factores que impiden su desarrollo. Es por ello el desarrollo de estudios para analizar los diversos factores tanto externos como internos inherentes al individuo y así prestar la 
debida atención a las implicaciones de políticas para sacar el mayor provecho posible a las potencialidades que ofrece el emprendedor.

Esta investigación tiene como objetivo describir y analizar los factores asociados a la decisión de los individuos de ser emprendedores en América Latina, basándose en los distintos enfoques y teorías del emprendimiento y mediante la aplicación de un modelo de regresión Logit por el método de máxima verosimilitud. La información utilizada proviene de la Encuesta de la Corporación Andina de Fomento (CAF) realizada en el año 2012 para una muestra de ciudades de Argentina, Bolivia, Brasil, Colombia, Ecuador, Perú, Uruguay, Venezuela y Panamá. La CAF realiza constantemente encuestas para diversos estudios y solamente en el año 2012 incluyó un modulo especial referente al emprendimiento.

\section{Esfera conceptual del emprendedor}

El primer indicio del concepto de emprendedor aparece en la obra de Joseph Alois Schumpeter de 1936 "The theory of economic development: an inquiry into profits, capital credit, interest, and business cycle". En este documento, el autor define al emprendedor como un individuo generador de crecimiento económico, y por lo tanto, bienestar en la sociedad (Schumpeter, 1936:153).

Debido a la existencia de varios enfoques en la investigación del emprendimiento, el término tiende a tener múltiples definiciones y teorías asociadas. El significado se remonta prácticamente al origen de la palabra en francés "entrepreneur" cuyo significado representa al "pionero" y utilizada inicialmente para referirse a los aventureros del "nuevo mundo" sin saber con certeza qué esperar en él, incertidumbre que hoy en día caracteriza al emprendedor (Herrera y Montoya, 2013:19). Con el tiempo, el término se hizo más generalizado para identificar a quien comenzaba una empresa o relacionado con cualquier persona decidida a llevar adelante un proyecto, incluyendo aquellos sin fines económicos (Formichella, 2004:3).

Sanyang y Huang (2009:321) argumentan que el emprendimiento descansa en el fortalecimiento de dos factores fundamentales: de las habilidades de los emprendedores y de las condiciones a las cuales se enfrentan. Estos dos factores no están aislados y por el contrario, están interconectados a través de políticas de incentivos. Para otros autores el emprendedor simplemente se hace $y$ no nace, entendiendo esa formación de emprendedores como interacciones y percepciones de procesos que permiten identificar las oportunidades de emprendimiento en el medio ambiente, y ayudan a la potenciación de los mismos (Krueguer y Brazeal, 1994:92; Know y Arenius, 2010:318).

Cuervo (2005:295) ofrece una definición más amplia. El emprendedor no es solo un individuo creador e identificador de una oportunidad de negocios, es quien decide crear riqueza y valor a los procesos productivos de pequeñas y medianas empresas 0 simplemente actúa como un vendedor de ideas para el desarrollo de un negocio. No solo es un coordinador o controlador de la actividad, también se anticipa a los cambios y realiza una continua evaluación en la manera de llevar una empresa y sus operaciones. Todo esto lo convierte en un actor permanente en la 
reinvención de los productos y procesos de la empresa en pro de establecer redes y nuevas oportunidades.

De esta manera y tomando en consideración los aspectos anteriores, el emprendedor puede ser definido como aquel individuo que identifica y desarrolla una idea de negocio creando riqueza y oportunidades para sí mismo y para otros al mismo tiempo.

\section{Caracterización del emprendedor}

Los estudios referentes al emprendimiento se enfocan en las características típicas para poder llegar a ser buenos emprendedores aunque investigaciones recientes señalan al emprendimiento como una forma de comportamiento sujeto de ser cambiado y aprendido, por lo tanto, la creatividad puede ser vista como un proceso o un producto (Herrera y Montoya, 2013:19). Algunas caracterizaciones pueden ser desarrolladas tomando en consideración las principales escuelas del pensamiento (clásica, neoclásica, schumpeteriana y austríaca), las cuales fueron resumidas por Herrera y Montoya (2013:20).

En la escuela clásica, predomina la caracterización de un individuo con preferencias al riesgo, lo cual le permite elevados retornos a la inversión, además de innovador y con suma inteligencia (Herrera y Montoya, 2013:20). Las ideas neoclásicas se enfocan más hacia un líder superior y carismático, con espontaneidad y capacidades innatas para adquirir conocimientos mediante la experiencia (Herrera y Montoya, 2013:21). Los neoclásicos aportaron muy poco a la definición y caracterización del emprendedor y al igual a los clásicos, no se llegó a un acuerdo en general. Sin embargo, investigadores como Jonh Bates Clark y Frank Knight diferían del resto en el sentido de considerar al emprendedor como no tomador de riesgos, debido a que éste los asegura o los transfiere (Herrera y Montoya, 2013:22).

La escuela de Schumpeter (cuyo máximo y único exponente es él mismo) caracteriza al emprendedor con aptitudes no comunes y solo están presentes en una pequeña porción de la población, cuya característica principal es el de la promoción de productos innovadores (Formiccella, 2004:5; Herrera y Montoya, 2013:19). En efecto, Schumpeter atribuye a la acción de los emprendedores como el principal mecanismo en el proceso del desarrollo económico y clave para corregir las distorsiones del sistema económico (Croitoru, 2012:141).

La escuela de pensamiento austríaca defiende el equilibrio que genera el emprendedor en el mercado al lograr mejorar y hacer más eficiente la red comercial y con ello, crear nuevas riquezas, lo cual lo convierte en un individuo en constante estado de alerta a las oportunidades. En resumen, es un complemento a los planteamientos de Schumpeter. Se entiende en esencia al emprendedor como un innovador, en virtud del desarrollo de conceptos novedosos e identifica las dimensiones críticas para luego asegurar la colaboración de expertos en la materia (Biais y Perrotti, 2008:1121).

Lanzas et al, (2009:269) realiza una clasificación del emprendedor en virtud de tres enfoques: económico, sociológico y psicológico. El primero se refiere a la naturaleza y las consecuencias de los emprendimientos en relación con el desarrollo de una industria y su directa incidencia en el 
crecimiento económico. El sociológico se basa en explicar el porqué hay regiones con mayor cultura de emprendimiento que otras, las condiciones del entorno, factores étnicos y de estructura familiar. El psicológico trata la relación entre personalidad y factores como competencias y experiencias.

EnSpencery Gómez(2008:1100), resalta una clasificación de tres tipos de emprendimiento desde la forma más simple a la forma más compleja. Primero se encuentra el auto-empleo como la forma más simple de emprendimiento, debido a que rara vez requiere de una inversión financiera importante, habilidad gerencial avanzada o un entendimiento legal sustancial. La segunda forma es la dirección de negocios pequeños, el cual requiere de un mayor grado de inversión, organización y de habilidades gerenciales para el sostenimiento de las operaciones diarias.

Finalmente, se encuentran los negocios nuevos, caracterizados por presencia en bolsas de valores $u$ otras estructuras de organización financiera y reflejan una forma avanzada de emprendimiento, con sofisticadas operaciones, incorporación legal mayor, lo cual despierta un atractivo interés de los inversores. No obstante, la presencia de cada tipo de emprendimiento dependerá de la dimensión institucional de los países en los cuales se encuentran inmersos.

En este orden de ideas, se puede caracterizar al emprendedor de la siguiente manera: individuo con poca aversión al riesgo lo cual le permite obtener el máximo valor esperado de retorno de la inversión, innovador con ideas diferenciadoras, optimista y positivo en cada decisión y sobre todo, oportunista debido a su capacidad de identificar nuevas opciones de emprendimiento. Para el innovador no existen fallas, solo eventos adversos minimizados mediante la toma de decisiones acertadas.

\section{Factores socio-culturales asociados al emprendimiento: Breve revisión de la literatura empírica}

La contribución de la actividad emprendedora a la sociedad puede variar de un país a otro. El ejercicio del emprendimiento puede ser en algunos casos productivos y en otros, de manera improductivo, cuya sustentación recae en la estructura de pagos de la economía o más formalmente, en las reglas de juego establecidas (Baumol, 1990:918; Douglas y Shepherd, 2002:85). El emprendimiento improductivo puede tomar formas como la evasión fiscal y los tomadores de rentas de los mercados imperfectos, hechos que dependen del sistema productivo ordinario.

Para una aproximación al emprendimiento improductivo, Wong et al (2005:344) proporciona evidencia empírica con datos del Global Entrepreneurship Monitor (GEM) para el año 2002 de una muestra de 37 países. Los autores no encuentran evidencia estadística suficiente para establecer la contribución al crecimiento de la economía de las actividades emprendedoras representada en nuevos negocios, cuya base explicativa se debe a la "actividad refugiada" o "actividad comercial", similar a lo ya señalado como actividad de subsistencia, lo cual carece o genera escaso crecimiento tecnológico.

Esta idea tiene también sustento en un trabajo similar de Velliere $y$ Peterson (2009:476). Utilizando datos 
de GEM de 44 países para los años 2004 y 2005, encuentran como en las economías emergentes el aporte de los emprendedores al crecimiento económico es atenuado, debido al sustento en una actividad por necesidad o por algún logro personal. En los países desarrollados el resultado es positivo, explicado mediante la explotación de los emprendedores del conocimiento y de las libertades ofrecidas por sus sistemas gubernamentales.

La imperfección de los mercados puede llevar a descubrimientos de nuevas oportunidades de negocios, lo cual otorga a los emprendedores la posibilidad de explotar esas ineficiencias (Cuervo, 2005:297). En este orden de ideas, Hvide (2009:1030), provee suficiente evidencia para determinar la información imperfecta de empresas acerca del valor de los empleados evidenciará mejores salarios con poca flexibilidad y así dar cabida a una mayor productividad y a la innovación. Asimismo, la imperfección de los mercados eleva los costos asociados a la investigación y a la capacidad investigadora, lo cual altera el espíritu empresarial y explicar la diferencia y frecuencia emprendedora en ciertas industrias, regiones 0 períodos de tiempo (Kerr et al, 2014:44).

El enfoque de la teoría del capital humano refuerza estos argumentos al apuntar el conocimiento de los individuos como factor potenciador de sus habilidades cognitivas $y$, permite elevar la productividad y eficiencia. Esto no solamente es el resultado de la educación formal, sino de la experiencia y del aprendizaje en el trabajo, además de la educación no formal que puede darse en ella (Davidsson y Honig, 2003:316). En efecto, estos autores evidencian a través de una regresión logística la relación positiva entre emprendimiento e indicadores de capital humano representados por años de educación, experiencia gerencial y años de experiencia laboral.

Formichella (2008:137), en un estudio sobre emprendedores en Argentina, encuentra impacto positivo del nivel educativo, la edad y el género masculino sobre la probabilidad de ser emprendedor, razón por la cual estas variables deben considerarse para el fomento al autoempleo productivo y así evitar su conversión a autoempleados de subsistencia.

Resultados similares presentan Freire y Teijeiro (2009:21) en un estudio para el año 2008 en la región de Galicia en España entre la población joven. Los resultados muestran que la edad y la dotación de capital humano son preponderantes en la decisión de ser emprendedor, la condición de tener pareja afecta negativamente a la probabilidad de ser emprendedor, y esto coincide con el atributo psicológico de ser independiente en el mercado laboral con la independencia del individuo a nivel social.

Bajo una perspectiva de género, Fuentes y Sánchez (2010:21) en un estudio para España, encuentran una menor iniciativa emprendedora en las intenciones de las mujeres que además, presentan en mayor medida el temor al fracaso como un obstáculo a la hora de pensar en la creación de una empresa propia, destacando en las mujeres la iniciativa, la creatividad y la autoconfianza como aspectos asociados al emprendimiento. Para los varones, en cambio, se relaciona con características como el deseo de enfrentarse a nuevos retos o el entusiasmo ante los proyectos.

En virtud de lo anterior, es preciso considerar los señalamientos de la Organización Internacional del Trabajo 
(OIT) en lo referente a los diversos obstáculos a los que se enfrentan las mujeres en casi todas las fases de sus actividades empresariales (similares al del sexo masculino) y a restricciones adicionales específicas de su género de origen sociocultural arraigadas, lo que constituye un proceso a largo plazo (Morales y Rahe, 2007:66).

En el estudio de García del Junco et al, (2007:969), igualmente para España, determinan el espíritu emprendedor asociado en la confianza en las personas que les rodean al crear la empresa, mentalidad universal $y$ compromiso con la sociedad, y tienen "obsesión" por satisfacer al cliente, en cambio, los de menor espíritu emprendedor, temen precipitarse en la toma de decisiones, poseen mentalidad localista, y no son propensos a arriesgar.

Moriano et al, (2006:88) realizaron un estudio para los estudiantes universitarios de la Comunidad Autónoma de Castilla y León en España. Encontraron que el género, la familia, la experiencia laboral, la educación hacia el autoempleo, el apoyo social, la percepción de barreras y los valores individualistas y colectivistas permiten predecir la intención de crear una empresa o trabajar por cuenta propia.

García y Wandosell (2004:21) por su parte, mediante un estudio empírico realzan las motivaciones y obstáculos para la creación de empresas en la región de Murcia en España. Entre las motivaciones se encuentran la realización socioeconómica, el espíritu inquieto y la posibilidad de aprovechar una oportunidad de negocio. Los principales obstáculos son las fuentes de financiamiento y los trámites asociados a la administración pública.

Otro resultado pueden contradecir los hallazgos comunes. Johansson (2000:134) evidencia para la economía finlandesa relación entre elevado nivel de riqueza con elevada probabilidad de emprender, mientras elevado nivel de educación se encuentra asociado con una menor probabilidad del emprendimiento.

En general, una parte de los diversos resultados puede ser explicada mediante lo denominado como el "estigma al fracaso" (Landier, 2006:2). Se puede observar en regiones como Europa y Asia, el estigma del fracaso está más arraigado y frecuentemente es mencionado como el mayor impedimento para el emprendimiento (Landier, 2006:11).

En consonancia con lo anterior, Mancilla y Amorós (2012:21) efectuaron un estudio para Chile para verificar cómo influye en individuos sin empresa, ciertas condiciones de un marco socio-cultural sobre la probabilidad para convertirse en emprendedores. Los resultados muestran principalmente que cuando un individuo percibe miedo social al fracaso, en cambio, su probabilidad de emprendimiento disminuye en presencia de role model (contacto directo con nuevos emprendedores) esa probabilidad aumentará.

La formación y la experiencia del empresario es clave en la supervivencia de la empresa, al observarse que estos aspectos reducen la probabilidad de cierre. Asimismo, la motivación para emprender parece tener una influencia significativa en la probabilidad de supervivencia de las empresas, lo cual evidencia diferencias entre los empresarios por necesidad y los empresarios por oportunidad (Simón et al, 2012:245)

\section{Metodología de la estimación empírica}

La información a utilizar proviene de la Encuesta de la Corporación Andina 
de Fomento (CAF) realizada entre el 21 de agosto y 19 de octubre del año 2012 y consta de 8.500 encuestas dirigidas a la población de hombres y mujeres entre 25 y 65 años de edad, con un margen de error del $4,9 \%$ para cada ciudad, con una confiabilidad estimada del $5 \%$, constituida para una muestra de ciudades de Argentina, Bolivia, Brasil, Colombia, Ecuador, Perú, Uruguay, Venezuela y Panamá, con un total de 1000 encuestas para cada país a excepción Panamá con 500. La encuesta incluye un módulo específico sobre las aptitudes para el emprendimiento, junto con otras series de variables sociodemográficas que permitirán categorizar a las personas involucradas en la actividad emprendedora.

La estimación empírica se realiza aplicando un modelo de regresión logit por el método de máxima verosimilitud. La aplicación de un modelo logit se justifica por la su gama aplicabilidad en el campo social mediante la utilización de variables discretas y categóricas, y supone que el logaritmo de la razón de probabilidades está relacionado linealmente con los parámetros (Silva y Barroso, 2004:43; Greene, 2006:787).

La variable dependiente se define como "Emprendedor" y toma el valor de 1 para aquel individuo que durante la aplicación de la encuesta se encontraba realizando una actividad emprendedora por cuenta propia (en calidad de jefe propio, dueño o socio de un negocio propio). Las variables explicativas la constituyen aquellas en relación directa con los factores socio-culturales del emprendimiento o que influyen en el espíritu emprendedor (capacidad para crear empresas): habilidad empresarial, tradición familiar, tolerancia el riesgo, autonomía, innovación y creatividad, e insatisfacción monetaria y laboral en los trabajos previos.
Asimismo, se establecen algunas otras variables que se han utilizado en Formichella (2008), Freire y Teijeiro (2009), Fuentes y Sánchez (2010), Mancilla y Amorós (2012) tales como sexo, educación, edad, edad al cuadrado, número de hijos, estado civil, nivel de educación y condición de nacional en el país de origen.

La variable "habilidad empresarial" se encuentra compuesta por el promedio simple de una serie de preguntas que tratan de medirla. Estas constituyen rasgos acerca de la personalidad del individuo constituidas por cuatro módulos: orientación al logro, locus de control interno, capacidad multitareas y autoeficacia.

La orientación al logro se vincula con emprendedores exitosos que buscan destacarse trazándose objetivos ambiciosos y llevando a los individuos a tomar responsabilidad por sus acciones y a buscar nuevas y mejores formas de actuar para mejorar los resultados. Para ello, se utilizan preguntas en las que se evalúa si los individuos prefieren actividades desafiantes en lugar de aquellas fáciles de resolver, o si no les importa realizar un trabajo rutinario o si les desagrada que las cosas no se hagan debidamente.

El locus de control interno está asociado con la creencia del individuo de sus logros dependen más de sus acciones o de factores externos, lo cual supone que individuos con esta característica tienen una mayor tendencia a establecer su propio negocio. La forma de medirla es preguntándole al individuo si lo que ha logrado en su vida depende de sus acciones, o si los malos resultados de su vida se debe a la mala suerte

Con respecto a la capacidad multitareas, ésta se asocia con las habilidades de realizar varias tareas a la 
vez y con la preferencia de tener un manejo aceptable de distintas capacidades. La medición de este atributo se ha realizado mediante una simple pregunta en la que el individuo debe contestar si prefiere ser relativamente bueno en varias tareas o muy bueno en unas pocas.

La autoeficacia consiste en la creencia del individuo en su propia habilidad para responder a los desafíos o para alcanzar ciertos objetivos propuestos, atributo relacionado con la confianza en sí mismos o el optimismo. Su medición se basa en preguntas donde el individuo responde si cumple las promesas que hace o si es capaz de aprender cualquier cosa si se lo propone.

La variable "tolerancia al riesgo" al igual de "habilidad empresarial", se encuentra medida a través de preguntas para indagar si el individuo debe tener toda la información necesaria antes de tomar una decisión o la preferencia de un empleo con un pago fijo seguro frente a otro con mayor remuneración pero con cierto grado de incertidumbre. Con esta variable se busca la característica fundamental del emprendedor como lo es su capacidad para tomar riesgos. Este atributo y su relación positiva con el emprendimiento ha sido corroborada en trabajos como los de Cramer et al, (2002) y Caliendo et al, (2009) (Citados en CAF, 2013:31).

La variable "autonomía" refleja un aspecto de la actividad emprendedora: ser su propio jefe o el deseo por tener más control sobre los objetivos. Se basa en indagar la personalidad a través de preguntas que analizan si el individuo se siente incómodo cuando otros deciden por él, o si en general tiende a defender su punto de vista cuando alguien está en desacuerdo con su posición.

La variable "tradición familiar" es una pregunta simple al determinar si algunos de los padres incursionaron en la actividad emprendedora, con la finalidad de establecer un vínculo familiar positivo con la idea del emprendimiento. La variable "innovación y creatividad" se define como una dimensión asociada con la gestión de un individuo para convertir nuevas ideas o tecnologías en productos a través del emprendimiento. Igualmente se indaga con dos preguntas para saber el nivel del individuo en este aspecto. Es de esperar mayor propensión al emprendimiento en individuos más creativos.

En relación a las variables "insatisfacción monetaria" e "insatisfacción laboral", se precisan a través de pregunta directa con una escala de insatisfacción de su situación monetaria y laboral. Trata de medir el grado de insatisfacción o de satisfacción del individuo en su actual situación laboral y su ganancia monetaria asociada. Mientras más insatisfecha se encuentra el individuo en estos aspectos, será más propenso a asumir riesgos mediante un emprendimiento.

La especificación econométrica se basa en un modelo logit para establecer relaciones entre un conjunto de factores socio-culturales asociados al emprendimiento. El modelo asume que las perturbaciones son homocedásticas y no correlacionadas, no adopta el supuesto de linealidad entre la variable dependiente (variable dicotómica) y las variables explicativas. En el modelo a desarrollar se han incluido variables tales como: tradición familiar empresarial, habilidad empresarial, tolerancia al riesgo, autonomía, innovación y creatividad e insatisfacción monetaria y laboral, género, edad como variable de experiencia, edad al cuadrado, condición de nacional o migrante en el país de origen, número de hijos, estado civil, nivel educativo. Una vez determinadas las variables, el modelo sigue la siguiente especificación: 


$$
\begin{gathered}
\mathrm{P}(\mathrm{Y}=1)=\ln \left(\frac{\mathrm{P}=\text { emprendedor }}{1-\mathrm{P}=\text { no emprendedor }}\right)=\alpha+\beta_{1}(\text { género })+\beta_{2}(\text { edad })+ \\
\beta_{3}\left(\text { edad }^{2}\right)+\beta_{4}(\text { nacional })+\beta_{5}(\text { hijos })+\beta_{6}(\text { estado civil })+\beta_{7}(\text { nivel educativo })+ \\
\beta_{8}(\text { tradición familiar empresarial })+\beta_{9}(\text { habilidad empresarial })+ \\
\beta_{10}(\text { tolerancia al riesgo })+\beta_{11}(\text { autonomía })+\beta_{12}(\text { innovación y creatividad })+ \\
\left.\left.\beta_{13} \text { (insatisfacción monetaria }\right)+\beta_{14} \text { (insatisfacción laboral }\right)+\varepsilon_{i}
\end{gathered}
$$

\begin{tabular}{|c|c|}
\hline Variable & Definición \\
\hline \multicolumn{2}{|r|}{ Dependiente } \\
\hline \multirow[t]{2}{*}{ Emprendedor } & 1 = Emprendedor \\
\hline & $0=$ No emprendedor \\
\hline \multicolumn{2}{|l|}{ Independientes } \\
\hline \multirow[t]{2}{*}{ Género } & 1 = Masculino \\
\hline & $0=$ Femenino \\
\hline Edad & Edad de la persona (numérica) \\
\hline Edad $^{2}$ & Edad de la persona al cuadrado (numérica) \\
\hline \multirow[t]{2}{*}{ Nacional } & 1 = Nacido en el país \\
\hline & $0=$ Nacido en otro país \\
\hline Hijos & Número de hijos (numérica) \\
\hline \multirow[t]{2}{*}{ Estado civil } & 1 = Casado o en unión \\
\hline & $0=$ Otro \\
\hline \multirow[t]{11}{*}{ Nivel educativo } & 1 Sin nivel educativo / sin instrucción \\
\hline & 2 Preescolar \\
\hline & 3 Básica / Primaria incompleta \\
\hline & 4 Básica / Primaria completa \\
\hline & 5 Media diversificada y profesional / Bachillerato incompleta \\
\hline & 6 Media diversificada y profesional / Bachillerato completa \\
\hline & 7 Técnica superior / Terciario no universitaria incompleta \\
\hline & 8 Técnica superior / Terciario no universitaria completa \\
\hline & 9 Universitaria incompleta \\
\hline & 10 Universitaria completa \\
\hline & 11 Especialización / Maestría / Doctorado \\
\hline
\end{tabular}

$\begin{array}{ll}\text { La variable dependiente } & \text { variables con C categorías y a cada } \\ \text { "emprendedor" y las variables } & \begin{array}{l}\text { categoría le corresponde un conjunto } \\ \text { de valores identificando dicha clase }\end{array} \\ \text { independientes género, nacional, estado } & \text { (ver Tabla 1). Algunos individuos de la } \\ \text { civil y tradición familiar empresarial son } & \text { muestra se han excluidos por presentar } \\ \text { variables dicotómicas, es decir toman } & \text { inconsistencias en la información (16 en } \\ \text { valores } 1 \text { o } 0 \text {. El resto son variables } & \text { total). }\end{array}$

Tabla 1

Variables utilizadas en el análisis de le regresión 


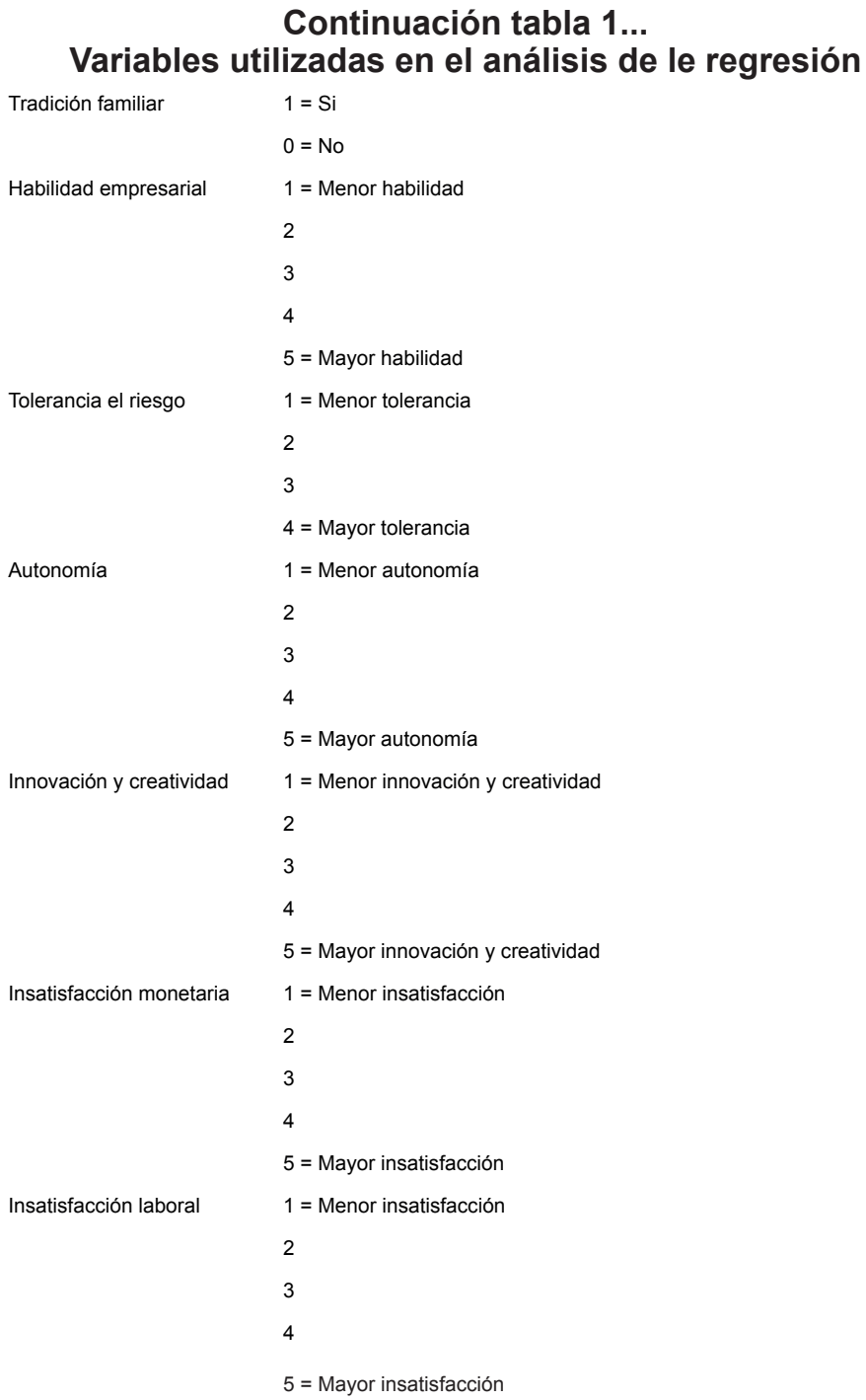

$5=$ Mayor insatisfacción

Fuente: Elaboración propia en base a la encuesta de la CAF

\section{Resultados y discusión}

Los estadísticos descriptivos de la muestra se muestran en Tabla 2. Brevemente se hará una descripción. Se puede apreciar mayor proporción de hombres emprendedores en relación a las mujeres (439 lo cual representa el 62,2\%). En el caso de los no emprendedores, esta situación se invierte $(46.9 \%)$. Por el lado de la condición de nacional o migrante, casi la totalidad de los emprendedores son de del país de origen y más de la mitad 
de los individuos son casados. Con respecto a la edad, se aprecia como los emprendedores en promedio tienen 40,9 años, y los individuos no emprendedores 41.8 años. La tradición familiar en los individuos emprendedores duplica en proporción al de los individuos no emprendedores, 31.9 frente a 15,2. Finalmente, en el resto de las variables resalta para los emprendedores en comparación con la muestra de los no emprendedores, mayor nivel educativo e insatisfacción monetaria.

Tabla 2

Estadísticos descriptivos para las variables seleccionadas

\begin{tabular}{|c|c|c|c|c|c|}
\hline & $\mathbf{N}$ & Media & Desv. Estándar & Min. Valor & $\begin{array}{l}\text { Máx. } \\
\text { Valor }\end{array}$ \\
\hline Emprendedor (Emp= 1) & 705 & & & & \\
\hline Género (Masculino = 1) & 439 & & & & \\
\hline Nacional & 667 & & & & \\
\hline Estado civil (casado o en unión = 1) & 400 & & & & \\
\hline Tradición familiar empresarial & 225 & & & & \\
\hline Edad & & 40,9 & 10,5 & 25,0 & 65,0 \\
\hline Hijos & & 2,2 & 1,7 & 0,0 & 12,0 \\
\hline Nivel educativo & & 7,0 & 2,4 & 1,0 & 11,0 \\
\hline Habilidad empresarial & & 3,9 & 0,4 & 2,7 & 5,0 \\
\hline Tolerancia el riesgo & & 2,9 & 1,1 & 1,0 & 4,0 \\
\hline Autonomía & & 3,8 & 0,6 & 1,7 & 5,0 \\
\hline Innovación y creatividad & & 3,4 & 0,9 & 1,0 & 5,0 \\
\hline Insatisfacción monetaria & & 4,4 & 0,8 & 1,0 & 5,0 \\
\hline Insatisfacción laboral & & 3,2 & 1,2 & 1,0 & 5,0 \\
\hline No emprendedor $(E m p=0)$ & 7.779 & & & & \\
\hline Género (Masculino = 1) & 3.655 & & & & \\
\hline Nacional & 7.622 & & & & \\
\hline Estado civil (casado o en unión $=1$ ) & 3.662 & & & & \\
\hline Tradición familiar empresarial & 1.195 & & & & \\
\hline Edad & & 41,8 & 11,7 & 25,0 & 65,0 \\
\hline Hijos & & 2,2 & 1,8 & 0,0 & 19,0 \\
\hline Nivel educativo & & 6,1 & 2,2 & 0,0 & 11,0 \\
\hline Habilidad empresarial & & 3,9 & 0,4 & 1,2 & 5,0 \\
\hline Tolerancia el riesgo & & 3,1 & 1,0 & 1,0 & 4,0 \\
\hline Autonomía & & 3,8 & 0,6 & 1,0 & 5,0 \\
\hline Innovación y creatividad & & 3,5 & 0,8 & 1,0 & 5,0 \\
\hline Insatisfacción monetaria & & 4,2 & 0,9 & 1,0 & 5,0 \\
\hline Insatisfacción laboral & & 3,4 & 1,2 & 1,0 & 5,0 \\
\hline Total & 8.484 & & & & \\
\hline
\end{tabular}

Fuente: Elaboración propia en base a la encuesta de la CAF. 
Factores socio-culturales asociados al emprendedor: evidencia empírica... Angel Enrique Martínez Gámez

En general, los trabajadores por cuenta propia poseen muy bajo nivel de productividad y capacidad de generación de ingresos (ligados al talento empresarial), lo cual implica no necesariamente la presencia de factores psicológicos ligados al individuo emprendedor. También han sido denominados trabajadores de subsistencia y si las habilidades empresariales pueden no estar presentes en ellos, tienen pocas perspectivas de expandir sus actividades y de generar empleo, y esto a su vez limita su capacidad de producción y de elevar los ingresos. Por estas razones, se excluyen de la estimación. Los resultados de la aplicación del modelo logit se muestra en la Tabla 3.

Tabla 3

Resultados modelo logit para establecer la relación entre Emprendimiento* $y$ factores socio culturales

\begin{tabular}{lccc}
\hline & Coeficientes & Std. Err. & Z \\
\hline Constante & $-1,5444$ & 0,1016 & $-1,54$ \\
Género & 0,1286 & 0,1016 & 1,27 \\
Edad & $-0,0692$ & 0,0374 & $-1,85$ \\
Edad ${ }^{2}$ & 0,0005 & 0,0004 & 1,28 \\
Nacional & $-0,4684$ & 0,3453 & $-1,36$ \\
Hijos & 0,0848 & 0,0305 & 2,78 \\
Estado civil & 0,3023 & 0,1010 & 2,99 \\
Nivel educativo & 0,2071 & 0,0229 & 9,01 \\
Tradición familiar empresarial & 0,4065 & 0,1117 & 3,64 \\
Habilidad empresarial & 0,3018 & 0,1435 & 2,10 \\
Tolerancia el riesgo & $-0,0326$ & 0,0460 & $-0,70$ \\
Autonomía & $-0,1423$ & 0,0947 & $-1,50$ \\
Innovación y creatividad & $-0,0372$ & 0,0600 & $-0,62$ \\
Insatisfacción monetaria & 0,3226 & 0,0622 & 5,18 \\
Insatisfacción laboral & $-0,1878$ & 0,0417 & $-4,50$ \\
& & & \\
N & 2.238 & & \\
LR chi2 (14) & 215,05 & & \\
Prob > chi2 & 0,0000 & & \\
Log likelihood & $-1.255,84$ & & \\
pseudo R2 & 0,0789 & & \\
\hline
\end{tabular}

* Excluyendo trabajadores por cuenta propia

La tabla muestra los resultados de la aplicación de un modelo de regresión Logit por el método de máxima verosimilitud, estimado en Stata 11.1.

Fuente: Elaboración propia 
Los resultados presentados en la Tabla 3 reflejan la ausencia de evidencia estadística de alguna relación entre emprendimiento y las variables asociadas como género, edad al cuadrado, condición de nacional en el país de origen, tolerancia al riesgo, autonomía y la innovación y creatividad. Con respecto a la edad, el coeficiente muestra relación positiva con la condición de ser emprendedor, es decir, las habilidades se incrementan con la experiencia e individuos de mayor edad pueden tener esa condición. Sin embargo, la variable edad al cuadrado (representa mayor propensión al riesgo) revela signo negativo aunque en menor medida, el cual sugiere disminución de preferencia por un emprendimiento en individuos de edad avanzada.

El signo positivo del nivel educativo refleja de manera cierta mayor educación asociado al emprendimiento. El cambio de signos en edad y edad al cuadrado refleja evidencia la existencia de una $U$ invertida en la relación. El número de hijos y la condición de casado u otra forma de unión revelan relación positiva y significativa con el emprendedor. Ambos resultados pueden ser explicados en virtud de quienes tienen mayores cargas familiares y comparten la responsabilidad del hogar con otra persona, tienen más margen de maniobra o estén más preparados para asumir riesgos.

El coeficiente positivo y significativo de la tradición familiar indica el hecho de ascendencia ligada al emprendimiento, lo cual estimula a las futuras generaciones a participar como emprendedores. La habilidad empresarial muestra signo positivo, tal como se esperaba de acuerdo a la caracterización del emprendedor el cual establece que en su mayoría poseen habilidades gerenciales

La insatisfacción monetaria resulta tener el efecto esperado, al presentar signo positivo, lo cual permite afirmar que las personas convertidas en emprendedoras suelen hacerlo también por motivos monetarios. No obstante, la insatisfacción laboral presenta signo negativo contrario a lo esperado, indicativo en América Latina de presencia de emprendedores cuya razón no necesariamente se encuentra ligada a la insatisfacción laboral en su trabajo anterior.

Es necesario hacer otro tipo de consideración para mejorar los resultados ya obtenidos. La figura del emprendedor suele no ser un elemento muy común en encuestas (sobre todo en muestras pequeñas), los resultados pueden estar sesgados dado a la subestimación de los parámetros estimados (King y Zeng, 2001). Para este caso, se propone un método denominado "logit ajustado para eventos extraños" ${ }^{\prime}$ el cual corrige la estimación del modelo logit cuando hay presencia de eventos extraños. Resalta este sesgo producido por la presencia de pocos " 1 ", disminuye cuando aumenta el tamaño de la muestra y, por el contrario, este sesgo aumenta al disminuir la muestra. Cuando la proporción de valores 1 está muy lejano a $0.50(p<0.50)$ el sesgo de estimación se amplía.

La muestra de la CAF no presenta pocos valores 1 , pero es conveniente eliminar este potencial sesgo, en virtud que su uso no afecta los resultados si el sesgo no estuviese presente. La Tabla 4 muestra el resultado junto con las probabilidades asociadas a la primeras

1 Es estimado en Stata mediante una aplicación desarrollada por Michael Tomz, Gary King y Langche Zeng de la Universidad de Harvard obtenido de la dirección web: http://gking.harvard. edu/scholar_software/relogit-rare-events-logistic-regression/1-1-stata 
Factores socio-culturales asociados al emprendedor: evidencia empírica... Angel Enrique Martínez Gámez

diferencia. Esta última no es más que el cambio en la probabilidad como una función de un cambio específico en una variable, siendo en este caso, del valor cero 0 a 1 , manteniendo el resto de las variables constantes en sus medias muestrales, expresado por la ecuación:

$\hat{\mathrm{y}}_{\mathrm{X}}=\mathrm{P}(\mathrm{Y}=1 \mid \mathrm{X}=1)-\mathrm{P}(\mathrm{Y}=1 \mid \mathrm{X}=0)$
Los valores obtenidos en el modelo logit de eventos extraños (Tabla 4) no difieren significativamente de los ya presentados en el logit anterior (Tabla 3 ), por lo tanto todas las consideraciones establecidas son válidas igualmente en estos resultados. A pesar de los ajustes, esto confirma las relaciones con cierto grado de robustez y pequeño sesgo.

Tabla 4

Resultados modelo logit para eventos extraños para establecer la relación entre Emprendimiento* y factores socio-culturales

\begin{tabular}{lcccc}
\hline & Coeficientes & Std. Err. & Z & $\begin{array}{c}\text { Primera } \\
\text { diferencia }\end{array}$ \\
\hline Constante & & & & \\
Género & $-1,5093$ & 0,9957 & $-1,52$ & 0,0255 \\
Edad & 0,1271 & 0,1013 & 1,25 & $-0,4921$ \\
Edad & $-0,0694$ & 0,0368 & $-1,88$ & 0,4345 \\
Nacional & 0,0005 & 0,0004 & 1,31 & $-0,1033$ \\
Hijos & $-0,4733$ & 0,3679 & $-1,29$ & 0,1414 \\
Estado civil & 0,0842 & 0,0315 & 2,67 & 0,0598 \\
Nivel educativo & 0,2997 & 0,1001 & 2,99 & 0,3695 \\
Tradición familiar empresarial & 0,2055 & 0,0230 & 8,90 & 0,0866 \\
Habilidad empresarial & 0,4043 & 0,1104 & 3,66 & 0,1138 \\
Tolerancia el riesgo & 0,3003 & 0,1408 & 2,13 & $-0,0191$ \\
Autonomía & $-0,0326$ & 0,0474 & $-0,69$ & $-0,0752$ \\
Innovación y creatividad & $-0,1413$ & 0,0923 & $-1,53$ & $-0,0275$ \\
Insatisfacción monetaria & $-0,0371$ & 0,0596 & $-0,62$ & 0,2109 \\
Insatisfacción laboral & 0,3192 & 0,0602 & 5,30 & $-0,1529$ \\
N & $-0,1865$ & 0,0416 & $-4,47$ & \\
\hline Excluyendo tabajadores & & & & \\
\hline & 2.238 & & & \\
\hline
\end{tabular}

* Excluyendo trabajadores por cuenta propia

La tabla muestra los resultados de la aplicación de un modelo de regresión Logit para eventos extraños por el método de máxima verosimilitud, estimado en Stata 11.1.

Fuente: Elaboración propia 
Con respecto a la primera diferencia individual de cada factor asociado, en el caso de la variable género al no tener significancia estadística, la probabilidad individual asociada refleja la misma probabilidad de emprender tanto en hombres como en mujeres, similar característica en cuanto a un individuo con la condición de nacional o extranjero. En edad, la probabilidad indica que por cada año adicional, la probabilidad de emprender disminuye en casi $50 \%$. Asimismo, un hijo adicional en el hogar también incrementa la probabilidad de emprender en $14,1 \%$ y un nivel educativo adicional alcanzado en $36,9 \%$.

En el caso de estar en unión de pareja, la probabilidad es baja, de apenas $6 \%$, mientras que tener ascendentes emprendedores de $8,6 \%$. La habilidad empresarial tiene una probabilidad individual de $11,3 \%$ y la insatisfacción monetaria y laboral de $21 \%$ y $-15,2 \%$, respectivamente. Las mayores probabilidades asociadas a la edad y el nivel educativo determinan el énfasis en políticas y planes de desarrollo que involucren a la población joven y la educación, en pro de aprovechar las potencialidades presentes en el emprendimiento.

\section{Conclusiones}

La esfera del emprendimiento debe disponer de un mayor debate y alcance teórico en los actuales momentos, debido al aporte de las estimaciones empíricas y su sustento para las discusiones. Las características de los emprendedores exitosos no necesariamente son las mismas de las personas con visiones a emprender, probablemente asociada en mayor medida a emprendimientos de mayor tamaño y no los de menores escalas, los cuales son los más característicos en América Latina.

Considerando el enfoque de los factores generadores y potenciadores, se puede considerar como ambos contribuyen y fomentan el espíritu emprendedor, no son elementos aislados y en algunos casos no se les concede la importancia necesaria para la consideración de políticas. El emprendedor es el resultado de interacciones sociales como la tradición de las personas de su propio círculo o de un sistema de casta, además de características propias como las habilidades empresariales, las cuales pueden ser adquiridas y potenciadas.

La complejidad al tratar de responder si el emprendedor se hace o nace, hace posible la combinación de diversos factores. La experiencia y formación pueden hacer al emprendedor junto con la combinación de rasgos psicológicos que intervienen en la sustentación y crecimiento de su actividad. La experiencia no es condicionante para emprender y en efecto, cada vez se evidencia mayores iniciativas de emprendimiento en la población joven. Si hay inclusión en la actividad emprendedora de jóvenes, estos presentan una serie de características y hábitos en proceso de asimilación o ya asimiladas desde edades muy tempranas, además de un claro indicativo de dinamismo y un interés por alternativas distintas a la esfera de un trabajador asalariado, lo cual puede ser beneficioso para las economías de la región.

Por otra parte, el nivel educativo representa un factor ligado a la actividad emprendedora, en virtud de que amplía los horizontes y pueden llegar a tener una visión más amplia de las 
oportunidades de negocios. Es así como un bajo nivel de educación aumentará el emprendimiento por necesidad (ligada más a los autoempleados o trabajadores por cuenta propia) y un mayor nivel de educación aumentará el emprendimiento por oportunidad (emprendedores con escalas de negocios más amplias y con empleados). Generalmente, la baja insatisfacción laboral en estos últimos confirma la presencia de emprendedores oportunos y no por necesidad.

No se puede establecer que la actividad emprendedora sea de mayor atención para los hombres o para las mujeres. El emprendimiento tanto en los hombres como en las mujeres se establece en el hecho de preferir otras alternativas laborales con mayor satisfacción como seguridad, flexibilidad laboral para atender obligaciones del núcleo familiar, alternativas de mayores ingresos, entre otros.

El fomento de la actividad emprendedora en los países de la región debe tomar en cuenta los distintos elementos establecidos. Es preciso un mayor apoyo a la cultura emprendedora mediante programas formativos en pro de minimizar los factores adversos a la hora de establecer emprendimientos y asimismo, de estructuras de seguimiento para evaluarla de manera permanente.

\section{Referencias bibliográficas}

Baumol, William (1990), Entrepreneurship: productive, unproductive, and destructive, Journal of Political Economy, Volumen 98, No 5, The University of Chicago Press, pp 893921.

Biais, Bruno y Perotti, Enrico (2008), Entrepreneurs and New Ideas, The RAND Journal of Economics, Volumen 39, $\mathrm{N}^{\circ} 4$, pp 1105-1125.

Corporación Andina de Fomento CAF (2012), Encuesta de hogares 2012. En http://scioteca.caf.com/ handle/123456789/410. Fecha de consulta: 04 de mayo de 2015.

Corporación Andina de Fomento CAF (2013), Emprendimientos en América Latina: desde la subsistencia hacia la transformación productiva, Reporte de Economía y Desarrollo. Bogotá, Colombia.

Croitoru, Alin (2012), Schumpeter, J.A., 1934 (2008), The theory of economic development: an inquiry into profits, capital, credit, interest and the business cycle, translated from the German by Redvers Opie, New Brunswick (U.S.A) and London (U.K.): transaction publishers, Journal of Comparative Research in Anthropology and Sociology, Volumen $3, \mathrm{~N}^{\circ} 2$, Romania, University of Bucharest, pp 1-13.

Cuervo, Alvaro (2005), Individual and Environmental Determinants of Entrepreneurship, The International Entrepreneurship and Management Journal, Volumen 1, $\mathrm{N}^{\circ} 3$, United States, pp 293-311.

Davidsson, Per y Honig, Benson (2003), The role of social and human capital among nascent entrepreneurs, Journal of Business Venturing, Volumen 18, № 3 , Australia, pp 301331.

Douglas, Evan J. y Shepherd, Dean (2002), Self-employment as a career choice: attitudes, entrepreneurial intentions, and utility maximization, Entrepreneurship Theory and Practice, Volumen 26, $N^{\circ} 3$, Australia, pp 81-90.

Formichella, María M. (2004), El concepto de emprendimiento y su relación con la educación, el empleo y el desarrollo local, Instituto Nacional de Tecnología Agropecuaria, Buenos Aires, Argentina.

Formichella, María M. (2008), Educación y trabajo independiente: un aporte a la evidencia empírica, Estudios de Economía Aplicada, Volumen 26, № 3, España, pp 115-139.

Freire S., María J. y Teijeiro Á. Mercedes (2009), Análisis de los factores que afectan a la decisión de ser emprendedor, Cuadernos de Economía. Volumen 32, № 90, España, pp 5-28. 
Fuentes, Fernando y Sánchez, Sandra (2010), Análisis del perfil emprendedor: una perspectiva de género, Estudios de Economía Aplicada, Volumen $28, \mathrm{~N}^{\circ}$ 3, España, pp 1-28.

García del Junco, Julio; Álvarez, Pedro y Reyna, Rafael (2007), Características del Emprendedor de Éxito en la Creación de PYMES Españolas, Estudios de Economía Aplicada, Volumen 25, No 3, España, pp 951974.

García, Antonio y Wandosell, Gonzalo (2004), Motivaciones y obstáculos en la creación de empresas: efectos de la experiencia empresarial, Boletín Económico del ICE, $\mathrm{N}^{\circ} 2819$, España, pp 19-36.

Greene, William (2006), Análisis Econométrico, Tercera Edición. Madrid: Prentice Hall.

Herrera, Carmen E. y Montoya, Luz A. (2013), El emprendedor: una aproximación a su definición y caracterización, Punto de Vista, Volumen IV, № 7, Colombia, pp 7-30.

Hvide, Hans K. (2009), The Quality of Entrepreneurs, The Economic Journal, Volumen 119, № 539, Royal Economic Society, pp 1010-1035.

Johansson, Edvard (2000), Self-employment and liquidity constraints: evidence from Finland, Scandinavian Journal of Economics, Volumen 102, $\mathrm{N}^{\circ} 1$, United Kingdom, pp 123-134.

Kerr, William; Nanda, Ramana y Rhodes-Kropf, Matthew (2014), Entrepreneurship as Experimentation, The Journal of Economic Perspectives, Volumen 18, $\mathrm{N}^{\circ}$ 3, American Economic Association, pp 25-48.

King, Gary y Zeng, Langche (2001), Logistic regression in rare events data, Political Analysis, Volumen $9, \mathrm{~N}^{\circ} 2$, pp 137-163.

Krueger, Norris y Brazeal, Deborah (1994). Entrepreneurial potential and potential entrepreneurs, Entrepreneurship Theory and Practice, $N^{\circ} 8$, Baylor University, pp 91-104.

Kwon, Seok-Woo y Arenius, Pia (2010), Nations of entrepreneurs: a social capital perspective, Journal of Business Venturing, $N^{\circ} 25,315-330$.
Landier, Augustin (2005), Entrepreneurship and the stigma of failure. Paper presentado a: MIT Finance, Development and Macro Workshops. En http://ssrn.com/ abstract $=850446$. Fecha de consulta: 21 de mayo de 2015.

Lanzas, Victoria; Lanzas, Francisco y Lanzas, Angela (2009), Propuesta para medir el perfil de los emprendedores de base tecnológica, Scientia et Technica, Año XV, № 43, Colombia, Universidad Tecnológica de Pereira, pp 267-272.

Mancilla, Claudio y Amorós, José E. (2012), La influencia de factores socio-culturales en el emprendimiento, evidencia en Chile 2007-2010, Multidisciplinary Business Review, Volumen 5 , № 1 , Chile, pp 14-25.

Morales, Carlos E. y Rahe, Martin (2007), Las competencias para el éxito de los emprendedores y de las emprendedoras, Harvard Deusto Business Review, № 157, pp 64-71.

Moriano, Juan A.; Palací, Francisco J. y Morales, José F. (2006), El perfil psicosocial del emprendedor universitario, Revista de Psicología del Trabajo y de las Organizaciones, Volumen 22, $\mathrm{N}^{\circ} 1$, Madrid, pp 75-99.

Sanyang, Saikou y Huang, Wen-Chi (2009), Entrepreneurship and economic development: the empretec showcase, International Entrepreneurship and Management Journal, Volumen 6, $N^{\circ} 3$, pp 317-329.

Schumpeter, Joseph A. (1936), The theory of economic development: an inquiry into profits, capital credit, interest, and business cycle, Cambridge, Harvard University Press.

Silva, Luis y Barroso, Isabel (2004), Regresión Logística. Cuadernos de Estadística 27, Editorial La Muralla, España.

Simón, Virginia; Revuelto, Lorenzo y Medina Aida (2012), La influencia de la formación, la experiencia y la motivación para emprender en la supervivencia de las empresas de nueva creación, Estudios Gerenciales, Volumen 28, Colombia, pp 237-262. 
Factores socio-culturales asociados al emprendedor: evidencia empírica...

Angel Enrique Martínez Gámez

Spencer, Jennifer y Gómez, Carolina (2004), The Relationship among national structures, economic factors, and domestic entrepreneurial activity: a multicountry study, Journal of Business Research, № 57 , pp 10981107.

Valliere, Dave y Peterson, Rein (2009), Entrepreneurship and economic growth: Evidence from emerging and developed countries, Entrepreneurship \& Regional Development, Volumen 21, $\mathrm{N}^{\circ} 5-6$, pp 459-480.

Wong, Poh; Ho, Yuen y Autio, Erkko (2005), Entrepreneurship, innovation and economic growth: evidence from GEM data, Small Business Economics, Volumen 24, № 3, pp 335- 350. 


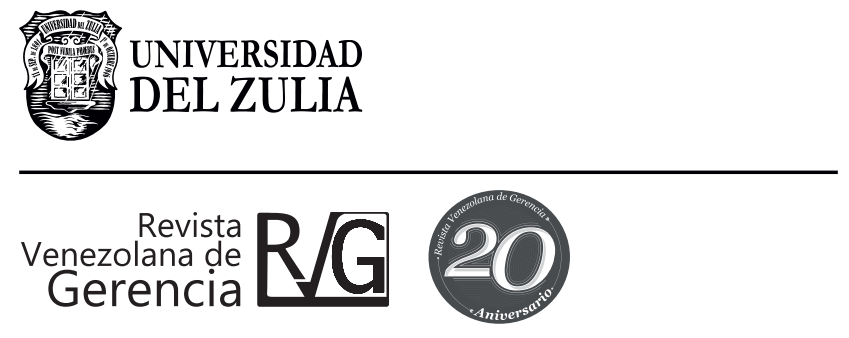

Año 21, No. 74

Esta revista fue editada en formato digital y publicada en junio del 2016, por la Universidad del Zulia, Vicerrectorado Académico, Serbiluz - Fondo editorial, Maracaibo -Venezuela. 